\title{
THE SPECTRAL LOCALIZATION PROPERTY FOR DIAGONAL OPERATORS AND SEMIGROUPS
}

\author{
N. K. NIKOLSKI
}

Dedicated to the 75th birthday of $V$. P. Havin, my university teacher and the only personality whose features I've been unable to exhaust since.

\section{§1. Introduction. The SPECTRAL LOCALIZATION PROPERTY}

Let $X$ be a Banach space, $\mathcal{X}=\left(x_{j}\right)$ a family of vectors in $X$, and $T$ a bounded linear operator on $\operatorname{span}_{X}(\mathcal{X})$ such that

$$
T x_{j}=\lambda_{j}(T) x_{j} \text { for all } j .
$$

In this case, we say that $\left(\lambda_{j}(T)\right)_{j}$ (as well as the operator $T$ itself) is a (Hadamard) multiplier for $\mathcal{X}$ (or an $\mathcal{X}$-multiplier). Denote by $\operatorname{Mult}(\mathcal{X})$ the set of all $\mathcal{X}$-multipliers. Clearly, $\operatorname{Mult}(\mathcal{X})$ endowed with the usual operator norm is a unital Banach algebra with respect to pointwise operations, and $\operatorname{Mult}(\mathcal{X}) \subset l^{\infty}$. A family $\mathcal{X}$ is said to have the spectral localization property (SLP) if

$$
\sigma(T)=\operatorname{clos}\left\{\lambda_{j}(T)\right\}
$$

for every multiplier $T \in \operatorname{Mult}(\mathcal{X})$. Clearly, the SLP is equivalent to the following inverse closedness property:

$$
\left.\left(T \in \operatorname{Mult}(\mathcal{X}),\left|\lambda_{j}(T)\right| \geq \delta>0 \text { for all } j\right) \Longrightarrow T^{-1} \text { is bounded (is in } \operatorname{Mult}(\mathcal{X})\right) .
$$

This property is of considerable interest from various points of view, and it was studied independently in different contexts. It is also important to know whether there exists an estimate for $\left\|T^{-1}\right\|$ in terms of the lower spectral parameter

$$
\delta_{T}=\inf _{j}\left|\lambda_{j}(T)\right| .
$$

The following quantity is responsible for this:

$$
c_{1}(\delta, M(\mathcal{X}))=\sup \left\{\left\|T^{-1}\right\|: T \in \operatorname{Mult}(\mathcal{X}),\|T\| \leq 1, \delta_{T} \geq \delta\right\},
$$

2010 Mathematics Subject Classification. Primary 42B15, 46B15, 47A10.

Key words and phrases. Hadamard multiplier, inverse closedness property, Bézout equations, multiplier corona problem, Muckenhoupt exponential basis.

The author was partially supported by the EU Marie Curie Action contract TODEQ and an ANR project DYNOP (France).

In particular, I am warmly grateful to Professors W. Arendt, Yu. Tomilov, and J. Zemanek for fruitful discussions during my stay in the IM PAN (Institute of Mathematics of the Polish Academy of Sciences) and the University of Torun, as well as for excellent working conditions offered to me by the TODEQ contract.

The paper took its final form during my research stay at MSU in East Lansing (2009). My sincere thanks are to Professor A. Volberg and the MSU Math Department for their hospitality. 
where $0<\delta \leq 1$. Similarly, we can consider the corresponding problem for joint inversions for columns $T^{\mathrm{col}}=\left(T_{k}\right)_{k=1}^{n} \in \operatorname{Mult}(\mathcal{X})^{n}$ of $n$ multipliers $T_{k}$, i.e., for the existence of a line $S \in \operatorname{Mult}(\mathcal{X})^{n}$ such that

$$
S T^{\mathrm{col}}=\sum_{k=1}^{n} S_{k} T_{k}=I d
$$

(an order $n$ Bézout equation; $n=1$ corresponds to the inversion problem). An obvious necessary condition is

$$
\delta_{T^{\mathrm{col}}}=\inf _{j}\left|\lambda_{j}(T)\right|>0,
$$

where $\left|\lambda_{j}(T)\right|=\left(\sum_{k=1}^{n}\left|\lambda_{j}\left(T_{k}\right)\right|^{2}\right)^{1 / 2}$. The following function gives a numerical measurement of this property:

$$
c_{n}(\delta, M(\mathcal{X}))=\sup \left\{\left(\inf \|S\|: S \in \operatorname{Mult}(\mathcal{X})^{n}, S T^{\mathrm{col}}=I d\right):\|T\| \leq 1, \delta_{T^{\mathrm{col}}} \geq \delta\right\},
$$

where $\|T\|=\left(\sum_{k=1}^{n}\left\|T_{k}\right\|^{2}\right)^{1 / 2}$. Being monotone nonincreasing, the functions $\delta \mapsto$ $c_{n}(\delta, M(\mathcal{X}))$ give rise to the following definition of critical constants $\delta_{n}=\delta_{n}(M(\mathcal{X}))$ of the algebra $\operatorname{Mult}(\mathcal{X})$ :

$$
\delta_{n}(M(\mathcal{X}))=\inf \left\{\delta: c_{n}(\delta, M(\mathcal{X}))<\infty\right\}=\sup \left\{\delta: c_{n}(\delta, M(\mathcal{X}))=\infty\right\},
$$

with an obvious convention for empty sets. We refer to [Ni2, Ni3, ENZ] for more about these functions and constants, as well as for some applications.

Clearly, $0 \leq \delta_{1} \leq \delta_{2} \leq \cdots \leq 1$. The relation $\delta_{n}=0$ implies that all Bézout equations of order at most $n$ satisfying the above necessary condition are solvable in $\operatorname{Mult}(\mathcal{X})$. The validity of the latter property for every $n=1,2, \ldots$ is equivalent to the fact that the obvious ("visible") complex homomorphisms $T \longmapsto \lambda_{j}(T), j \in J$, are dense in the space $\mathfrak{M}(\operatorname{Mult}(\mathcal{X}))$ of maximal ideals of $\operatorname{Mult}(\mathcal{X})$. The question on the density of $J$ in $\mathfrak{M}(\operatorname{Mult}(\mathcal{X}))$ is usually referred to as a multiplier corona problem.

We end this Introduction with an abridged list of known general facts about Hadamard multipliers; see [Si] for some of them.

(P1) It is well known (and easy) that

$$
\operatorname{Mult}(\mathcal{X})=l^{\infty}
$$

if and only if $\mathcal{X}$ is an unconditional basic sequence (and the indicator multipliers $\chi_{\sigma}$ correspond to the coordinate projections $\left.T=P_{\sigma}, P_{\sigma}\left(\sum a_{j} x_{j}\right)=\sum_{j \in \sigma} a_{j} x_{j}\right)$. Obviously, in this case, $\mathcal{X}$ has the SLP.

(P2) In harmonic analysis, say, in the one-variable classical harmonic analysis, the multipliers are known as Hadamard (Fourier) multipliers: here $\mathcal{X}=\mathcal{E}=\left(e^{i n x}\right)_{n \in \mathbb{Z}}$ and $X$ is a function (or distribution) space on the torus $\mathbb{T}=\{z: z \in \mathbb{C},|z|=1\}$.

For $X=L^{p}(\mathbb{T})$ and $p=1, \infty$, a sequence $\left(\lambda_{n}\right)$ is a (Hadamard) multiplier if and only if the $\lambda_{n}$ are the Fourier coefficients of a complex measure on $\mathbb{T}$. So, denoting by $\mathcal{M}(\mathbb{T})$ the space of all such measures (in fact, this is a convolution Banach algebra) and by $\mathcal{F}$ the (discrete) Fourier transformation, we can write

$$
\operatorname{Mult}\left(\mathcal{E}, L^{1}(\mathbb{T})\right)=\mathcal{F} M(\mathbb{T}) .
$$

The Wiener-Pitt-Schreider theorem says that in this case $\mathcal{E} \notin$ (SLP) (moreover, there exists a measure with real Fourier coefficients satisfying, say, $\widehat{\mu}(n) \geq 1$ for all $n \in \mathbb{Z}$ and 
such that $(1 / \widehat{\mu}(n))_{n}$ is not a multiplier). See $[\mathbf{R}$, GMcG] for a proof and for references to original sources. Moreover, it is worth mentioning that for this case, we can give some narrow bounds for the critical constants, namely,

$$
\frac{1}{2} \leq \delta_{n}\left(\operatorname{Mult}\left(\mathcal{E}, L^{1}(\mathbb{T})\right)\right) \leq \frac{1}{\sqrt{2}}
$$

for every $n=1,2, \ldots$ (see [Ni2, ENZ]). In particular, every multiplier $(\widehat{\mu}(j))_{j \in \mathbb{Z}}$ of $\mathcal{E}, L^{1}(\mathbb{T})$ with $1 / \sqrt{2}<\inf _{j \in \mathbb{Z}}|\widehat{\mu}(j)| \leq\|\mu\| \leq 1$ is invertible in $\operatorname{Mult}\left(\mathcal{E}, L^{1}(\mathbb{T})\right)$ but this is already not the case for $1 / 2 \leq \inf _{j \in \mathbb{Z}}|\widehat{\mu}(j)| \leq\|\mu\| \leq 1$.

For $p=2, \mathcal{E}$ is an orthonormal sequence, so $\operatorname{Mult}(\mathcal{E})=l^{\infty}$.

For $1<p<\infty, p \neq 2$, it is also known that $\mathcal{E} \notin$ (SLP) (S. Igari; M. Zafran); see $\mathrm{GMcG}$ for the proof and references.

(P3) It is known (and easy to show) that $\mathcal{X}$ is a (Schauder) basic sequence (not necessarily unconditional) if and only if

$$
b v \subset \operatorname{Mult}(\mathcal{X}),
$$

where $b v$ stands for the space of sequences of bounded variation:

$$
\left(\lambda_{j}\right) \in b v \Longleftrightarrow \sum_{j}\left|\lambda_{j}-\lambda_{j+1}\right|<\infty .
$$

It is also easily seen that in the case where $b v=\operatorname{Mult}(\mathcal{X}), \mathcal{X}$ has the SLP. For example, this is the case for $X=b v, \mathcal{X}$ being the standard $0-1$ basis in $b v$. Later on, we shall show that there exists no Hilbert space sequence $\mathcal{X}$ such that $\operatorname{Mult}(\mathcal{X})=b v$.

The paper is organized as follows. In $\S 2$ we introduce the spectral localization problem for Hilbert space Schauder bases.

$\S 3$ contains constructions of Muckenhoupt exponential bases without the SLP on weighted $L^{2}(\mathbb{T}, w)$ spaces.

In $\S 4$ we deal with a comparison of the SLP and its quantitative version (the " $\delta_{1}=0$ problem"). In particular, we show that there exist sequences $\mathcal{X}$ in Banach spaces that have the SLP but do NOT satisfy $\delta_{1}=0$. To the contrary, it is shown that for a Schauder basis in a Hilbert space, the SLP always implies $\delta_{1}=0$. An example is given of an asymptotically orthogonal Schauder basis with $\delta_{1}=1$.

$\S 5$ contains a discussion of several close-to-being-open questions, in particular, the SLP for $C_{0}$-semigroups. We give an example of a $C_{0}$-semigroup on a Hilbert space that is diagonal with respect to a summation basis and has no SLP.

\section{§2. Introduction. The SLP for Hilbert space Bases}

So, by (P1), an unconditional basic sequence satisfies the SLP, but, in general, for merely a (Schauder) basic sequence, this is not the case. However, the examples from (P2) and (P3) above concern bases in non-Hilbert spaces only. A natural question is, how is it for bases in a Hilbert space? Below we give an example of a Hilbert space basis without the SLP. To the contrary, we do not know of any Hilbert space Schauder basis having SLP that is not an unconditional basis (so, not equivalent to an orthogonal one).

First, it should be mentioned that, in fact, there exist quite a few concrete examples of Hilbert space bases that are not unconditional (cf. [Si]). Essentially, the only general source for such bases is the following Hunt-Muckenhoupt-Wheeden theorem: the sequence of exponentials

$$
\mathcal{E}=\left\{e^{i n x}: n \in \mathbb{Z}\right\}
$$


is a basis in a weighted space $L^{2}(\mathbb{T}, w)$,

$$
L^{2}(\mathbb{T}, w)=\left\{f: \int_{\mathbb{T}}|f(z)|^{2} w(z) d m(z)<\infty\right\},
$$

where $w$ stands for a nonnegative measurable function on $\mathbb{T}$ and $m$ for the normalized Lebesgue measure, if and only if the following Muckenhoupt $\left(A_{2}\right)$ condition is fulfilled:

$$
A_{2}(w)=\sup _{I}\left(\frac{1}{|I|} \int_{I} w d m\right) \cdot\left(\frac{1}{|I|} \int_{I} \frac{1}{w} d m\right)<\infty,
$$

where $I$ runs over all $\operatorname{arcs} I \subset \mathbb{T}$. It is easily seen that $\mathcal{E}$ is an unconditional basis (equivalent to an orthonormal one) if and only if $0<\inf _{z} w(z)$ and $\sup _{z} w(z)<\infty$ (we mean the essential inf and sup). A typical example of an $\left(A_{2}\right)$ weight (whose properties were known much earlier than the general criteria) is

$$
w(z)=\frac{1}{|1-z|^{\beta}}, \quad-1<\beta<1 .
$$

The corresponding theory of weighted $L^{p}(\mathbb{T}, w)$ spaces is well developed (see $[\mathrm{D}, \mathrm{St}, \mathrm{T}$, Ni1]).

In what follows, we mostly focus our attention on the trigonometric system

$$
\mathcal{E}=\left\{e^{i n x}: n \in \mathbb{Z}\right\}
$$

in $L^{2}$ spaces

$$
L^{2}(\mathbb{T}, \mu)=\left\{f: \int_{\mathbb{T}}|f(z)|^{2} d \mu(z)<\infty\right\},
$$

where $\mu$ is a nonnegative (finite) measure on $\mathbb{T}$, and we use the shortened notation $M(\mu)$ for the space of multipliers $\operatorname{Mult}(\mathcal{E})$ over the space $L^{2}(\mathbb{T}, \mu)$. In the case of $\mu$ having a density $w \in L^{1}(\mathbb{T})$ with respect to the normalized Lebesgue measure $m$ (i.e., for $\mu=w m$ ), we write $L^{2}(w)$ for $L^{2}(\mathbb{T}, \mu)$ and $M(w)$ for $M(w m)$. From (P3) above, it follows that $w \in\left(A_{2}\right)$ if and only if $b v \subset M(w)$.

Recall also that the basis constant of a sequence $\mathcal{X}=\left(x_{j}\right)$ is

$$
b(\mathcal{X})=\sup _{m, n}\left\|P_{m, n}\right\|
$$

where $P_{m, n}$ stands for a coordinate projection,

$$
P_{m, n}\left(\sum c_{j} x_{j}\right)=\sum_{m \leq j \leq n} c_{j} x_{j}
$$

(defined initially on finite combinations of $x_{j}$ only). Then, $\mathcal{X}$ is a Schauder basis if and only if $b(\mathcal{X})<\infty$.

\section{§3. A Muckenhoupt basis without the SLP}

The following observation is the key point of our construction.

3.1. Lemma. Let $\nu$ be a nonnegative measure on $\mathbb{T}$, and let $w \in L^{1}(\mathbb{T})$. Then $\operatorname{Mult}(w) \subset$ $\operatorname{Mult}(w * \nu)$ and $T \in \operatorname{Mult}(w) \Longrightarrow\|T\|_{M(w * \nu)} \leq\|T\|_{M(w)}$. 
Proof. For a trigonometric polynomial $f$, we put $f_{\alpha}(z)=f(\bar{\alpha} z)$. Let $T=\left(t_{k}\right) \in \operatorname{Mult}(w)$ and denote $\|T\|=\|T\|_{M(w)}$. Then

$$
\begin{aligned}
\|T f\|_{w * \nu}^{2} & =\int_{\mathbb{T}}|T f(z)|^{2}(w * \nu)(z) d m=\int_{\mathbb{T}}|T f(z)|^{2} \int_{\mathbb{T}} w(\bar{\alpha} z) d \nu(\alpha) d m \\
& =\int_{\mathbb{T}} d \nu(\alpha) \int_{\mathbb{T}}|T f(z)|^{2} w(\bar{\alpha} z) d m=\int_{\mathbb{T}} d \nu(\alpha) \int_{\mathbb{T}}\left|(T f)_{\bar{\alpha}}(z)\right|^{2} w(z) d m \\
& =\int_{\mathbb{T}} d \nu(\alpha) \int_{\mathbb{T}}\left|\left(T f_{\bar{\alpha}}\right)(z)\right|^{2} w(z) d m=\int_{\mathbb{T}}\left\|T f_{\bar{\alpha}}\right\|_{w}^{2} d \nu(\alpha) \leq\|T\|^{2} \int_{\mathbb{T}}\left\|f_{\bar{\alpha}}\right\|_{w}^{2} d \nu(\alpha) \\
& =\|T\|^{2} \int_{\mathbb{T}} d \nu(\alpha) \int_{\mathbb{T}}|f(\alpha z)|^{2} w(z) d m=\|T\|^{2} \int_{\mathbb{T}} d \nu(\alpha) \int_{\mathbb{T}}|f(z)|^{2} w(\bar{\alpha} z) d m \\
& =\|T\|^{2} \int_{\mathbb{T}}|f(z)|^{2}(w * \nu)(z) d m=\|T\|^{2}\|f\|_{w * \nu}^{2} .
\end{aligned}
$$

Therefore, $T \in \operatorname{Mult}(w * \nu)$.

The following fact is known in the theory of $\left(A_{2}\right)$ weights, but the proofs there are much more involved.

3.2. Corollary. If $w \in\left(A_{2}\right)$ and $\nu$ is a nonnegative measure on $\mathbb{T}$, then $w * \nu \in\left(A_{2}\right)$. The basis constant of $\mathcal{E}$ in $L^{2}(w * \nu)$ does not exceed that constant in $L^{2}(w)$.

Indeed, by Lemma 3.1, the inclusion $b v \subset M(w)$ implies $b v \subset M(w * \nu)$. The corresponding norm inequality also follows.

3.3. Lemma. Let $\lambda$ be a nonnegative measure on $\mathbb{T}$, and let $\alpha \in \mathbb{T}$. The rotation operator $T_{\alpha} f=f(\alpha z)$ is bounded on $L^{2}(\mathbb{T}, \lambda)$ (i.e., $\left(\alpha^{k}\right)_{k \in \mathbb{Z}} \in M(\lambda)$ ) if and only if

$$
\sup _{A} \frac{\lambda_{\alpha}(A)}{\lambda(A)}<\infty
$$

where

$$
\lambda_{\alpha}(A)=\lambda(\bar{\alpha} A)
$$

and $A$ runs over all measurable subsets of $\mathbb{T}$ with $\lambda(A)>0$. Moreover,

$$
\left\|T_{\alpha}^{n}\right\|=\sup _{A}\left(\frac{\lambda_{\alpha^{n}}(A)}{\lambda(A)}\right)^{1 / 2}
$$

for every $n \geq 1$.

Proof. Given a trigonometric polynomial $f$, we have

$$
\left\|T_{\alpha}^{n} f\right\|_{\lambda}^{2}=\int_{\mathbb{T}}\left|f\left(\alpha^{n} z\right)\right|^{2} d \mu=\int_{\mathbb{T}}|f(z)|^{2} d \lambda_{\alpha^{n}} .
$$

Therefore, $T_{\alpha}$ is bounded if and only if $\int_{\mathbb{T}}|f(z)|^{2} d \lambda_{\alpha} \leq C \int_{\mathbb{T}}|f(z)|^{2} d \lambda$ for every continuous function $f$, the latter being equivalent to $\lambda_{\alpha}(A) \leq C \lambda(A)$ for every $A$. The result follows.

The next two lemmas are inspired by the fact that a rotation operator $T_{\alpha} f(z)=f(\alpha z)$ on the space $L^{2}(\nu)$, where $\nu=\sum_{k \geq 0} a_{k} \delta_{\alpha^{k}}$, is simply the backward shift on $l^{2}\left(a_{k}\right)=$ $\left\{\left(f\left(\alpha^{k}\right)_{k \geq 0}: \sum\left|f\left(\alpha^{k}\right)\right|^{2} a_{k}<\infty\right\}=L^{2}(\nu)\right.$, and hence $\sigma\left(T_{\alpha} \mid L^{2}(\nu)\right)=\overline{\mathbb{D}}$.

3.4. Lemma. Let $\mu$ be a nonnegative measure on $\mathbb{T}$, and let $\lambda=\mu * \nu$, where

$$
\nu=\sum_{k \geq 0} a_{k} \delta_{\alpha^{k}}
$$


and $a_{k}>0,\left(a_{k} / a_{k+1}\right) \downarrow 1, \sum a_{k}<\infty, \delta_{\alpha^{k}}$ being the Dirac $\delta$-measure at $\alpha^{k}, \alpha \in \mathbb{T}$. Then $T_{\alpha}=\left(\alpha^{k}\right)_{k \in \mathbb{Z}} \in M(\lambda)$, and moreover,

$$
\left\|T_{\alpha}^{n}\right\| \leq\left(\frac{a_{0}}{a_{n}}\right)^{1 / 2}
$$

for every $n \geq 0\left(\|\cdot\|\right.$ means the operator norm over $\left.L^{2}(\mathbb{T}, \lambda)\right)$.

Proof. We apply Lemma 3.3 to the measure $\lambda=\mu * \nu=\sum_{k \geq 0} a_{k} \mu_{\alpha^{k}}$. Given a measurable $A \subset \mathbb{T}$ and an integer $n \geq 1$, we have

$$
\begin{aligned}
\lambda_{\alpha^{n}}(A) & =\sum_{k \geq 0} a_{k} \mu\left(\bar{\alpha}^{k+n} A\right)=\sum_{k \geq 0} \frac{a_{k}}{a_{k+n}} a_{k+} n \mu\left(\bar{\alpha}^{k+n} A\right) \\
& \leq \frac{a_{0}}{a_{n}} \sum_{k \geq 0} a_{k+n} \mu\left(\bar{\alpha}^{k+n} A\right) \leq \frac{a_{0}}{a_{n}} \lambda(A),
\end{aligned}
$$

which implies the result (Lemma 3.3).

3.5. Lemma. Let $\alpha, \mu, \nu$ and $\lambda=\mu * \nu=\sum_{k \geq 0} a_{k} \mu_{\alpha^{k}}$ be the same as in Lemma 3.4, and assume that for every $\epsilon>0$ and $N \in \mathbb{N}$ there exists $B \subset \mathbb{T}$ such that

$$
\max _{0<|k|<N} \mu\left(\alpha^{k} B\right)<\epsilon \mu(B)
$$

and $\mu\left(\bar{\alpha}^{k} B\right) \leq \mu(B)$ for every $k \geq 0$. Then

$$
\left\|T_{\alpha}^{n}\right\|=\left(\frac{a_{0}}{a_{n}}\right)^{1 / 2}
$$

for every $n \geq 1\left(\|\cdot\|\right.$ means the operator norm over $\left.L^{2}(\mathbb{T}, \lambda)\right)$.

In particular, all conditions are fulfilled for any $\alpha \in \mathbb{T}$ that is not a root of unity, and for $\mu=w m$, where $w(z)=|1-z|^{-\beta}, 0<\beta<1$ (as $B$, one can take a tiny arc centered at 1$)$.

Proof. By Lemma 3.4, we only need to check the inequality $\left\|T_{\alpha}^{n}\right\| \geq\left(\frac{a_{0}}{a_{n}}\right)^{1 / 2}$.

Let $\epsilon>0$, and let $B$ be a set from the hypothesis of the lemma, $A=\alpha^{n} B$. Then

$$
\begin{aligned}
\lambda(A) & =\sum_{k \geq 0} a_{k} \mu\left(\bar{\alpha}^{k} A\right) \\
& =a_{n} \mu\left(\bar{\alpha}^{n} A\right)+\sum_{0 \leq k<n} a_{k} \mu\left(\bar{\alpha}^{k} A\right)+\sum_{n<k<N} a_{k} \mu\left(\bar{\alpha}^{k} A\right)+\sum_{k \geq N} a_{k} \mu\left(\bar{\alpha}^{k} A\right) \\
& =a_{n} \mu(B)+\sum_{0 \leq k<n} a_{k} \mu\left(\alpha^{n-k} B\right)+\sum_{n<k<N} a_{k} \mu\left(\bar{\alpha}^{k-n} B\right)+\sum_{k \geq N} a_{k} \mu\left(\bar{\alpha}^{k-n} B\right) \\
& \leq a_{n} \mu(B)+\epsilon \mu(B) \sum_{0 \leq k<n} a_{k}+\epsilon \mu(B) \sum_{n<k<N} a_{k}+\sum_{k \geq N} a_{k} \mu(B) .
\end{aligned}
$$

Now, assuming that $c=\sum_{k \geq 0} a_{k}$ and $N$ is so large that $\sum_{k \geq N} a_{k}<\epsilon$, we get

$$
\lambda(A) \leq \mu(B)\left(a_{n}+2 \epsilon c+\epsilon\right) .
$$

On the other hand, $\lambda_{\alpha^{n}}(A)=\sum_{k \geq 0} a_{k} \mu\left(\bar{\alpha}^{k+n} A\right) \geq a_{0} \mu\left(\bar{\alpha}^{n} A\right)=a_{0} \mu(B)$, so that

$$
\left\|T_{\alpha}^{n}\right\|=\sup _{A}\left(\frac{\lambda_{\alpha^{n}}(A)}{\lambda(A)}\right)^{1 / 2} \geq\left(\frac{a_{0} \mu(B)}{\mu(B)\left(a_{n}+2 \epsilon c+\epsilon\right)}\right)^{1 / 2} .
$$

Letting $\epsilon$ tend to 0 , we obtain $\left\|T_{\alpha}^{n}\right\| \geq\left(\frac{a_{0}}{a_{n}}\right)^{1 / 2}$.

For $0<\beta<1$, let $w_{\beta}(z)=|1-z|^{-\beta}, z \in \mathbb{T}$. 
3.6. Theorem. Let $\alpha, \mu$ and $\nu$ be the same as in Lemma 3.5, and let $\lambda=\mu * \nu=$ $\sum_{k \geq 0} a_{k} \mu_{\alpha^{k}}$.

1) $T_{\alpha}=\left(\alpha^{k}\right)_{k \in \mathbb{Z}} \in M(\lambda)$, and moreover,

$$
\left\|T_{\alpha}^{n}\right\|_{M(\lambda)}=\left(\frac{a_{0}}{a_{n}}\right)^{1 / 2}
$$

for every $n \geq 0$.

2) If $\alpha=e^{2 \pi i \theta}$ with an irrational $\theta$, then $T_{\alpha}$ is not left invertible, and in particular, $\left(1 / \alpha^{k}\right)_{k} \notin M(w)$.

Moreover, if under the hypotheses of Lemma 3.5 the sets $B$ can be chosen to have an arbitrarily small diameter, then the spectrum of $T_{\alpha}$ (as an operator on $L^{2}(\lambda)$ ) is the closed unit disk $\overline{\mathbb{D}}$.

3) If, in addition to 2), $\mu=W m$ and $W \in\left(A_{2}\right)$, then the eigenfunctions of $T_{\alpha}$ (the exponentials $\mathcal{E}=\left(z^{k}\right)_{k \in \mathbb{Z}}$ ) form a Schauder basis in $L^{2}(\lambda), \lambda=w m, w=W * \nu$.

4) In particular, all hypotheses of 2) and 3) are fulfilled for $W=w_{\beta}$, which therefore gives an example of a Muckenhoupt weight $w$,

$$
w(z)=\sum_{k \geq 0} a_{k} w_{\beta}\left(\bar{\alpha}^{k} z\right)
$$

where $a_{k}>0,\left(a_{k} / a_{k+1}\right) \downarrow 1$ and $\sum a_{k}<\infty$, and a multiplier $T_{\alpha}=\left(\alpha^{k}\right)_{k \in \mathbb{Z}} \in M(w)$ such that $\sigma\left(T_{\alpha}\right)=\overline{\mathbb{D}}$.

Proof. All assertions were already proved in the lemmas, except for the spectrum properties in 2).

First, we show that $0 \in \sigma\left(T_{\alpha}\right)$, that is, $T_{\alpha}$ is not invertible. For this, it suffices to show that $\inf _{\|f\|=1}\left\|T_{\alpha} f\right\|=0$, and by Lemma 3.3 that the ratio $\lambda(A) / \lambda(\bar{\alpha} A)$ is not bounded over all measurable $A \subset \mathbb{T}$ such that $\lambda(A)>0$. By the hypothesis of Lemma 3.5 , there exist subsets $B_{n} \subset \mathbb{T}, n=1,2, \ldots$, such that $\mu\left(\bar{\alpha}^{k} B_{n}\right)=o\left(\mu\left(B_{n}\right)\right)$ as $n \longrightarrow \infty$ for every $k=1,2, \ldots$, and $\mu\left(\bar{\alpha}^{k} B_{n}\right) \leq \mu\left(B_{n}\right)$. This implies that

$$
\begin{aligned}
\frac{\lambda\left(\bar{\alpha} B_{n}\right)}{\lambda\left(B_{n}\right)} & =\frac{\sum_{k \geq 0} a_{k} \mu\left(\bar{\alpha}^{k+1} B_{n}\right)}{\sum_{k \geq 0} a_{k} \mu\left(\bar{\alpha}^{k} B_{n}\right)} \\
& \leq \frac{\sum_{k \geq 0} a_{k} \mu\left(\bar{\alpha}^{k+1} B_{n}\right)}{a_{0} \mu\left(B_{n}\right)}=\sum_{k \geq 0} \frac{a_{k} \mu\left(\bar{\alpha}^{k+1} B_{n}\right)}{a_{0} \mu\left(B_{n}\right)}=o(1)
\end{aligned}
$$

as $n \longrightarrow \infty$ (in view of the dominated convergence theorem). The inclusion $0 \in \sigma\left(T_{\alpha}\right)$ follows.

It remains to show that, in fact,

$$
\inf _{\|f\|_{\lambda=1}}\left\|f \zeta-T_{\alpha} f\right\|_{\lambda}=0
$$

for every $\zeta \in \mathbb{D}$. To this end we take a large $M \in \mathbb{N}$ and a set $B$ from the hypothesis of Lemma 3.5 whose diameter is so small that the sets $\alpha^{k} B, k=0,1, \ldots, M$, are pairwise disjoint. Now, put

$$
f=\sum_{k=0}^{M} \zeta^{k} \chi_{\alpha^{k} B}
$$

We have $\|f\|_{\lambda} \geq\left\|\chi_{B}\right\|_{\lambda} \geq\left(a_{0} \mu B\right)^{1 / 2}$, and, on the other hand, $T_{\alpha} \chi_{\alpha^{k} B}=\chi_{\alpha^{k-1} B}$, whence

$$
f \zeta-T_{\alpha} f=\sum_{k=0}^{M} \zeta^{k+1} \chi_{\alpha^{k} B}-\sum_{k=0}^{M} \zeta^{k} T_{\alpha} \chi_{\alpha^{k} B}=\zeta^{M+1} \chi_{\alpha^{M} B}-\chi_{\bar{\alpha} B} .
$$


This implies that

$$
\left\|f \zeta-T_{\alpha} f\right\|_{\lambda} \leq\left\|\zeta^{M+1} \chi_{\alpha^{M} B}\right\|_{\lambda}+\left\|\chi_{\bar{\alpha} B}\right\|_{\lambda}=|\zeta|^{M+1}\left(\lambda\left(\alpha^{M} B\right)\right)^{1 / 2}+(\lambda(\bar{\alpha} B))^{1 / 2} .
$$

We estimate these quantities in the same way as in Lemma 3.5. Namely,

$$
\begin{aligned}
\lambda(\bar{\alpha} B)=\sum_{k \geq 0} a_{k} \mu\left(\bar{\alpha}^{k+1} B\right) & =\sum_{0 \leq k<N-1} a_{k} \mu\left(\bar{\alpha}^{k+1} B\right)+\sum_{k \geq N-1} a_{k} \mu\left(\bar{\alpha}^{k+1} B\right) \\
& <\epsilon \mu(B) \sum_{0 \leq k<N-1} a_{k}+\sum_{k \geq N-1} a_{k} \mu(B) .
\end{aligned}
$$

Taking $B=B_{n}$ for $\epsilon=1 / n$, we get $\lambda\left(\bar{\alpha} B_{n}\right)=o\left(\mu\left(B_{n}\right)\right)$ as $n \longrightarrow \infty$. In order to make the quantity $|\zeta|^{M+1}\left(\lambda\left(\alpha^{M} B_{n}\right)\right)^{1 / 2}$ small, we simply choose $M=M_{n}$ so large that

$$
\frac{|\zeta|^{M+1}\left(\lambda\left(\alpha^{M} B_{n}\right)\right)^{1 / 2}}{\left(a_{0} \mu B_{n}\right)^{1 / 2}} \leq \frac{|\zeta|^{M+1}(\lambda(\mathbb{T}))^{1 / 2}}{\left(a_{0} \mu B_{n}\right)^{1 / 2}}=o(1) .
$$

Therefore, for $f=f_{n}$ (where $B=B_{n}, M=M_{n}$ ), we have

$$
\frac{\left\|f_{n} \zeta-T_{\alpha} f_{n}\right\|_{\lambda}}{\left\|f_{n}\right\|_{\lambda}} \leq \frac{\left(\lambda\left(\bar{\alpha} B_{n}\right)\right)^{1 / 2}+|\zeta|^{M_{n}+1}(\lambda(\mathbb{T}))^{1 / 2}}{\left(a_{0} \mu B_{n}\right)^{1 / 2}}=o(1)
$$

as $n \longrightarrow \infty$. This implies that $\zeta \in \sigma\left(T_{\alpha}\right)$.

In the next section, we discuss the question of the numerical value of the critical constant for the above exponential basis. In particular, we show that a slight modification of the previous construction will give an "extremely bad" Hilbert space basis $\mathcal{X}$ having $\delta_{1}(\operatorname{Mult}(\mathcal{X}))=1$.

\section{$\S 4$. InVERSE ClOSEDNESS AND THE $\delta_{1}=0$ PROBLEM}

The "inverse closedness" of $\operatorname{Mult}(\mathcal{X})$ means that $\left(\lambda_{j}\right) \in \operatorname{Mult}(\mathcal{X})$ and $\inf _{j}\left|\lambda_{j}\right|>0$ imply $\left(1 / \lambda_{j}\right) \in \operatorname{Mult}(\mathcal{X})$. Of course, this is yet another way to say that $\operatorname{Mult}(\mathcal{X})$ obeys the SLP. We show that, for a Schauder basis $\mathcal{X}$ in a Hilbert space, the inverse closedness does imply that $\delta_{1}(\mathcal{X})=0$. On the other hand, we exhibit an example which shows that this is not the case for general sequences $\mathcal{X}$ in a Banach space, so that the SLP is compatible with $\delta_{1}(\mathcal{X})>0$. We start with an example of a Banach algebra of multipliers for a Banach space basis that has the SLP and $\delta_{1}(\mathcal{X})>0$.

4.1. Example: the algebra of "small $L^{p}$ multipliers" combines the SLP with $\delta_{1}>0$. Let $1<p<\infty, p \neq 2$, and let $m_{p}$ stand for the algebra of all "small Hadamard $L^{p}$ multipliers", that is, sequences of the form $\lambda_{j}=\lambda+\lambda_{j}^{\prime}, j \in \mathbb{Z}$, where $\lambda \in \mathbb{C}$ and $\left(\lambda_{j}^{\prime}\right)$ belongs to the norm closure in $\operatorname{Mult}\left(\mathcal{E}, L^{p}(\mathbb{T})\right)$ of the set of compactly supported sequences. Then the algebra $m_{p}$ satisfies the following properties:

1) $\mathcal{E}$ is a basic sequence in $m_{p}$ (a Schauder basis in its hull);

2) $m_{p}$ is a subalgebra of $\operatorname{Mult}\left(\mathcal{E}, L^{p}\right)$ satisfying the SLP;

3) $\delta_{1}\left(m_{p}\right)>0$.

Indeed, it is clear that $m_{p}$ is a unital (commutative) Banach algebra with respect to the pointwise multiplication $\left(\lambda_{j}\right) \cdot\left(\mu_{j}\right)=\left(\lambda_{j} \mu_{j}\right)$, and it is well known that the maximal ideal space of $m_{p}$ is $\mathbb{Z} \cup\{\infty\}$ (see GMcG]). Hence, $m_{p}$ obeys the SLP,

$$
\sigma(T)=\operatorname{clos}\left(\lambda_{j}(T)\right)=\left\{\lambda_{j}(T): n \in \mathbb{Z}\right\} \cup\left\{\lim _{j} \lambda_{j}(T)\right\}
$$

for every multiplier $T \in m_{p}$.

We can also easily show that $\mathcal{E}$ is a Schauder basic sequence in $m_{p}$. To this end, denote by $P_{m, n}$ the coordinate projection, $P_{m, n}\left(x_{j}\right)=y$, where $y_{j}=x_{j}$ for $m \leq j \leq n$ and 
$y_{j}=0$ otherwise. Then, for every multiplier $T \in \operatorname{Mult}\left(\mathcal{E}, L^{p}(\mathbb{T})\right)$ and every $f \in L^{p}(\mathbb{T})$, we have

$$
\left\|P_{m, n}(T) f\right\|_{L^{p}}=\left\|T P_{m, n}(f)\right\|_{L^{p}} \leq\|T\|_{\text {Mult }}\left\|P_{m, n}(f)\right\|_{L^{p}} \leq C\|T\|_{\text {Mult }}\|f\|_{L^{p}},
$$

where $C$ is the basis constant for $\mathcal{E}$ in $L^{p}(\mathbb{T})$, which shows that $\left\|P_{m, n}(T)\right\|_{\text {Mult }} \leq$ $C\|T\|_{\text {Mult }}$. Restricted to $m_{p}$, this estimate implies that $\mathcal{E} \cup\{1\}$ is a Schauder basic sequence.

On the other hand, $\delta_{1}\left(m_{p}\right)>0$ due to Igari-Zafran's result quoted in $\S 1$. Indeed, if we suppose that $\delta_{1}\left(m_{p}\right)=0$, then given a multiplier $T=\left(\lambda_{j}\right) \in \operatorname{Mult}\left(\mathcal{E}, L^{p}(\mathbb{T})\right)$ with $0<\delta=\inf _{j}\left|\lambda_{j}\right| \leq\|T\|_{\text {Mult }} \leq 1$, we can find a sequence $\left(T_{n}\right)$ of "small multipliers" strongly converging to $T$ and having the same spectral bounds, as follows:

$$
\lambda_{j}\left(T_{n}\right)=\lambda_{j} \text { for }|j| \leq n, \text { and } \lambda_{j}\left(T_{n}\right)=1 \text { elsewhere. }
$$

From the preceding paragraph it follows that $\sup _{n}\left\|T_{n}\right\| \leq 1+2 C$ and, of course, $\delta \leq$ $\left|\lambda_{j}\left(T_{n}\right)\right|$ for every $j, n$. Since $\delta_{1}\left(m_{p}\right)=0$, there exists a constant $A>0$ such that $\left\|T_{n}^{-1}\right\| \leq A$ for all $n$. Since $T_{n}^{-1} f=T^{-1} f$ for every trigonometric polynomial of degree at most $n$, we conclude that $T^{-1}$ is bounded, and hence $T$ is invertible in $\operatorname{Mult}\left(\mathcal{E}, L^{p}(\mathbb{T})\right)$, which contradicts the Igari-Zafran result.

The above example is not quite satisfactory because $m_{p}$ is a multiplier space for no sequences: the space $\operatorname{Mult}(\mathcal{X})$ is always closed for the WOT topology in the algebra $L(X)$ of all bounded operators, whereas $m_{p}$ is not. In the next example, the sequence $\mathcal{X}$ is very far from being a basis, but it allows $\operatorname{Mult}(\mathcal{X})$ to have the SLP and the property $\delta_{1}(\mathcal{X})>0$.

4.2. Example: a Banach space sequence $\mathcal{X}$ having $\operatorname{SLP}$ and $\delta_{1}(\mathcal{X})=1 / 2$. Let $\sigma=\left(\lambda_{j}\right)_{j \geq 1}$ be a dense subset in the disk $\mathbb{D}$, and let $\mathcal{X}=\left(x_{j}\right)$, where the $x_{j}(z)=$ $\left(1-\lambda_{j} z\right)^{-1}$ are regarded as functions in the space

$$
c_{0 a}=\left\{f=\sum_{k \geq 0} \hat{f}(k) z^{k}: \hat{f}(k)=o(1) \text { as } k \longrightarrow \infty\right\}
$$

endowed with the uniform norm $\|f\|=\sup _{k \geq 0}|\widehat{f}(k)|$. Then, the multiplier space $\operatorname{Mult}(\mathcal{X})$ is the trace on $\sigma$ of the Wiener algebra

$$
W=\left\{f=\sum_{k \geq 0} \hat{f}(k) z^{k}: \sum_{k \geq 0}|\hat{f}(k)|<\infty\right\},
$$

and hence

1) $\operatorname{Mult}(\mathcal{X})$ is inverse closed;

2) $\delta_{1}(\mathcal{X})=1 / 2$.

Indeed, the dual space of $c_{0 a}$, with respect to the bilinear pairing

$$
\langle f, g\rangle=\sum_{k \geq 0} \hat{f}(k) \widehat{g}(k)
$$

is $W$, and hence an operator $T x_{j}=\mu_{j} x_{j}, j \geq 1$, is a multiplier if and only if $T^{*}$ is a bounded operator on $W$ such that $T^{*} g\left(\lambda_{j}\right)=\mu_{j} g\left(\lambda_{j}\right)$ for every $g \in W$ and every $j \geq 1$. Taking $f=T^{*} 1 \in W$, we get $\mu_{j}=f\left(\lambda_{j}\right)$ for every $j$. Clearly, this is an algebra isomorphism of $\operatorname{Mult}(\mathcal{X})$ onto $W$. It is also clear that $\delta_{T}=\inf _{j}\left|\mu_{j}\right|=\inf _{z \in \mathbb{D}}|f(z)|$, which reduces the above properties 1) and 2) to similar properties for $W$. But it is well known that the algebra $W$ is inverse closed (Wiener's $1 / f$ theorem) and $\delta_{1}(W)=1 / 2$ (see [Ni2] or [ENZ]).

Without the SLP, we can give an example of a summation basis in a Hilbert space with a value of $\delta_{1}$ prescribed in advance, as follows. 
4.3. Example: a summation basis having a value of $\delta_{1}$ prescribed in advance. This example is borrowed from [NV].

Theorem ([NV]). Let $0 \leq \delta_{1} \leq 1$. There exists a sequence $\mathcal{X}=\left\{x_{j}\right\}_{j \geq 1}$ in a Hilbert space $H$ satisfying the following properties.

1. $\mathcal{X}$ is a minimal sequence and a summation basis: there exist a numerical matrix $A=$ $\left(a_{i j}: j \leq i\right)$ such that for every $x \in H$ there is a unique series $\sum_{j>1} c_{j} x_{j} A$-summable to $x$, in the sense that $x=\lim _{n} A_{n} x$, where $A_{n} x=\sum_{j=1}^{n} a_{n j} c_{j} x_{j}$, and moreover, $\left\|A_{n}\right\| \leq 1$ for every $n \geq 1$.

2. $\mathcal{X}=\left\{x_{j}\right\}_{j \geq 1}$ is block-orthogonal in the sense that there exists a sequence of integers $n_{1}=1<n_{2}<\cdots<n_{k}<\cdots$ such that the subspaces $\operatorname{span}\left(x_{j}: j \in\left[n_{k}, n_{k+1}\right)\right)$ are pairwise orthogonal.

3. $\delta_{1}(\mathcal{X})=\delta_{1}$.

The proof, for which we refer the reader to [NV], depends on a careful analysis of the restriction algebras $H^{\infty} \mid \sigma$ on special Blaschke subsets $\sigma \subset \mathbb{D}$. The construction shows that $\mathcal{X}$ does not satisfy the SLP.

It should be mentioned that, in fact, the result quoted is a refinement of the following one (the main theorem from GMN]).

Theorem (GMN]). Let $H^{2}$ be the Hardy space on the unit disk $\mathbb{D}$ and $\mathcal{X}=\left(x_{j}\right)_{j \geq 1}$ a family of reproducing kernels

$$
x_{j}(z)=\frac{1}{1-\bar{\lambda}_{j} z}, \quad z \in \mathbb{D}, \quad \lambda_{j} \in \mathbb{D} .
$$

The following statements are equivalent:

1) $\mathcal{X} \in S L P$

2) $\delta_{1}(\mathcal{X})=0$

3) the algebra $\operatorname{Mult}(\mathcal{X})$ has no corona;

4) $\left(\lambda_{j}\right)$ satisfies the following weak embedding property (WEP): for every $\epsilon>0$ there exists a number $C>0$ such that

$$
\inf _{j}\left|b_{\lambda_{j}}(z)\right|>\epsilon \Longrightarrow \sum_{j} \frac{\left(1-|z|^{2}\right)\left(1-\left|\lambda_{j}\right|^{2}\right.}{\left|1-\bar{\lambda}_{j} z\right|^{2}} \leq C,
$$

where $b_{\lambda}(z)=\frac{z-\lambda}{1-\bar{\lambda} z}$ stands for a Blaschke factor.

In particular, finite unions of interpolating sequences satisfy the WEP.

4.4. Example: for every $\epsilon>0$ there exists a Muckenhoupt weight $w$ such that the exponentials $\mathcal{E}=\left(z^{k}\right)_{k \in \mathbb{Z}}$ in a space $L^{2}(w)$ satisfy the following:

(a) $b(\mathcal{E})<1+\epsilon$;

(b) $\delta_{1}(\mathcal{E})>1-\epsilon$.

Recall that always $b(\mathcal{E}) \geq 1(b(\mathcal{E})$ denotes the basis constant of $\mathcal{E}$; see the Introduction), and the relation $b(\mathcal{E})=1$ means (for a sequence in a Hilbert space) that $\mathcal{E}$ is an orthogonal basic sequence. Also, property (b) above means that there exists $T \in \operatorname{Mult}(\mathcal{E})$ such that $1-\epsilon<\inf _{k}\left|\lambda_{k}(T)\right| \leq\|T\| \leq 1$ but $T^{-1} \notin \operatorname{Mult}(\mathcal{E})$. On the other hand, a stronger condition, namely, $1=\left|\lambda_{k}(T)\right| \leq\|T\|=1$ for every $k$ and $\lambda_{k} \neq \lambda_{j}$, implies already that $\mathcal{E}$ is an orthogonal basic sequence (and, hence, has the SLP).

In order to construct an example mentioned in Subsection 4.4, we use Theorem 3.6. It follows that, given a sequence $\left(a_{k}\right)_{k \geq 0}$ satisfying the hypotheses of the theorem, we have a noninvertible multiplier $T$ such that $\left|\lambda_{j}(T)\right|=1$ for every $j \in \mathbb{Z}$ and $\|T\|=\left(a_{0} / a_{1}\right)^{1 / 2}$. Notice that the latter value is as close to 1 as we want, and the basis constant of $\mathcal{E}$ in 
the space $L^{2}(w), w=w_{0} * \nu$, does not exceed the same constant for $\mathcal{E}$ in $L^{2}\left(w_{0}\right)$ (see Lemma 3.1). Using the above multiplier, we obtain

$$
\delta_{1}\left(\mathcal{E}_{A}\right) \geq\left(a_{1} / a_{0}\right)^{1 / 2}>1-\epsilon
$$

for a convenient choice of $\left(a_{k}\right)_{k \geq 0}$.

Getting the estimate for (a), we recall that for the system of exponentials $\mathcal{E}$ in a space $L^{2}\left(w_{0}\right)$ the basis constant coincides with the norm of the Riesz projection,

$$
b(\mathcal{E})=\left\|P_{+}\right\|,
$$

where $P_{+}\left(\sum_{j} c_{j} z^{j}\right)=\sum_{j \geq 0} c_{j} z^{j}$ (see [Ni1, vol. 1, pp. 97-98]). It remains to apply the following lemma, where one can set $w^{1}=w_{0}=|1-z|^{-\beta}, 0<\beta<1$ (as in the last claim of Theorem 3.6).

4.4.1. Lemma. Suppose $w^{1} \in\left(A_{2}\right)$ and $0<\epsilon \leq 1$. Then $w^{\epsilon} \in\left(A_{2}\right)$ and

$$
\lim _{\epsilon \longrightarrow 0}\left\|P_{+}\right\|_{L^{2}\left(w^{\epsilon}\right) \longrightarrow L^{2}\left(w^{\epsilon}\right)}=1 .
$$

Proof. We cannot quote any known source for this lemma (see the references on the Riesz projection and the Hilbert transform in [Ni1, vol. 1]), since people interested in singular integrals are usually interested in the case where $A_{2}(w) \longrightarrow \infty$. The latter entails that in all known ready-to-apply upper estimates for $\left\|P_{+}\right\|_{L^{2}(w) \longrightarrow L^{2}(w)}$, there is a nonevaluated multiplicative constant. So, for the reader's convenience, we present here a modified argument from [Ni1, vol. 1, pp. 100-102], making use of the Helson-Szegö form of the basis criterion (which preceded, as is well known, the Hunt-MuckenhouptWheeden form).

Writing $w$ in the Helson-Szegö form, $w^{1}=e^{u+\widetilde{v}}$, where $u, v \in L^{\infty}(\mathbb{T}), \widetilde{v}$ stands for the harmonic conjugate, and $\|v\|_{\infty}<\pi / 2$, we observe that $\lim _{\epsilon \rightarrow 0}\left\|e^{ \pm \epsilon u}\right\|_{\infty}=1$. This shows that we can assume $u=0$. Now, the function $h=e^{(i v+\widetilde{v}) / 2}$ is an outer $H^{2}$ function such that $w^{\epsilon}=\left|h^{\epsilon}\right|^{2}, 0<\epsilon \leq 1$, and

$$
\left\|P_{+}\right\|_{L^{2}\left(w^{\epsilon}\right) \longrightarrow L^{2}\left(w^{\epsilon}\right)}^{2}=\left(1-\operatorname{dist}_{L^{\infty}}^{2}\left(\frac{\bar{h}^{\epsilon}}{h^{\epsilon}}, H^{\infty}\right)\right)^{-1}
$$

(see [Ni1, vol. 1, pp. 96, 101]). Moreover,

$$
\operatorname{dist}_{L^{\infty}}\left(\frac{\bar{h}^{\epsilon}}{h^{\epsilon}}, H^{\infty}\right) \leq\left\|\frac{\bar{h}^{\epsilon}}{h^{\epsilon}}-1\right\|_{\infty}=\left\|e^{-i \epsilon v}-1\right\|_{\infty} \longrightarrow 0
$$

as $\epsilon \longrightarrow 0$. The claim follows.

4.5. Example: an asymptotically orthogonal Hilbert space basis having $\delta_{1}=1$. We mean that there exists a basis $\mathcal{X}$ in a Hilbert space $H$ such that $\delta_{1}(\mathcal{X})=1$ and

$$
\lim _{m \longrightarrow \infty} \sup _{n>m}\left\|P_{m, n}\right\|=1
$$

(see the Introduction for the notation).

The construction we need follows from Example 4.4. Namely, using Example 4.4, we get

1) a family of sequences $A(n)=\left(a_{k n}\right)_{k \geq 0}$ and the corresponding weights $w=w_{A(n)}$ such that, for the basis $\mathcal{E}=\left(z^{k}\right)_{k \in \mathbb{Z}}$ in a space $L^{2}\left(w_{A(n)}\right)$ (we denote this basis by $\mathcal{E}_{n}$ ), we have $b\left(\mathcal{E}_{n}\right) \longrightarrow 1$ as $n \longrightarrow \infty$;

2) a noninvertible multiplier $T_{n}$ on $L^{2}\left(w_{A(n)}\right)$ such that $\left|\lambda_{j}\left(T_{n}\right)\right|=1$ for every $j \in \mathbb{Z}$, and $\left\|T_{n}\right\|=\left(a_{0 n} / a_{1 n}\right)^{1 / 2}$. 
We can choose the sequences in such a way that $\lim _{n}\left(a_{0 n} / a_{1 n}\right)=1$, which implies (for the same reason as in Example 4.4) that $\delta_{1}\left(\mathcal{E}_{n}\right) \longrightarrow 1$. Next, the basis property of $\mathcal{E}_{n}$ entails that, for every $n \geq 1$ and $m \geq 1$, we have

$$
\lim _{k} \epsilon(n, m, k)=0 \text {, }
$$

where

$$
\epsilon(n, m, k)=\inf \left\{\left\|T_{n} x\right\|:\|x\|=1, x \in P_{m, k} L^{2}\left(w_{A(n)}\right)\right\}=0
$$

and $\left.P_{m, k}\left(\sum_{j} c_{j} z^{j}\right)=\sum_{m \leq|j|<k} c_{j} z^{j}\right)$. By induction, we can find a sequence of integers $0=k_{0}<k_{1}<\cdots$ such that

$$
\lim _{n} \epsilon\left(n, k_{n-1}, k_{n}\right)=0
$$

Now, we consider the orthogonal sum of finite-dimensional spaces $P_{k_{n-1}, k_{n}} L^{2}\left(w_{A(n)}\right)$ and the multipliers $T_{n}$. Namely, we define the desired sequence $\mathcal{X}$ as a piecewise assemblage of sequences,

$$
\mathcal{X}=\sum_{n \geq 1} \oplus P_{k_{n-1}, k_{n}} \mathcal{E}_{n} \text { in the space } H=\sum_{n \geq 1} \oplus P_{k_{n-1}, k_{n}} L^{2}\left(w_{A(n)}\right) .
$$

The preceding observations show that $\mathcal{X}$ is a Schauder basis and that

$$
\lim _{m \longrightarrow \infty} \sup _{n>m}\left\|P_{m, n}\right\|=1
$$

(because $b\left(\mathcal{E}_{n}\right) \longrightarrow 1$ as $\left.n \longrightarrow \infty\right)$. Moreover, the multipliers $\widetilde{T}_{j}$, defined by

$$
\begin{aligned}
& \widetilde{T}_{j}=i d \text { on } P_{k_{n-1}, k_{n}} L^{2}\left(w_{A(n)}\right) \text { for } n \neq j, \\
& \widetilde{T}_{j}=T_{n} \text { on } P_{k_{n-1}, k_{n}} L^{2}\left(w_{A(n)}\right) \text { for } n=j,
\end{aligned}
$$

satisfy the properties $\left\|\widetilde{T}_{j}\right\| \leq\left(a_{0 j} / a_{1 j}\right)^{1 / 2}, \lim _{j}\left\|\widetilde{T}_{j}^{-1}\right\|=\infty$, and $\lambda_{i}\left(\widetilde{T}_{j}\right)=\lambda_{i}\left(T_{j}\right)$ for $k_{j-1} \leq|i|<k_{j}$ and $\lambda_{i}\left(\widetilde{T}_{j}\right)=1$ elsewhere. Since $\lim _{j}\left(a_{0 j} / a_{1 j}\right)=1$, this implies that $\delta_{1}(\mathcal{X})=1$.

The following theorem shows that a phenomenon similar to Example 4.2 is impossible for Schauder bases in a Hilbert space.

4.6. Theorem. Let $\mathcal{X}=\left\{x_{j}\right\}_{j \geq 1}$ be a basic sequence in a Hilbert space $H$ that satisfies $S L P$. Then $\delta_{1}(\mathcal{X})=0$, that is, $c_{1}(\delta$, $\operatorname{Mult}(\mathcal{X}))<\infty$ for every $\delta>0$.

The proof depends on the following Cotlar-Stein almost orthogonality lemma; see, for example, [Ni1, vol. 2, pp. 174-175] (we state it here in a simpler form based on the Schur boundedness test; see [Ni1, vol. 1, p. 282]).

Lemma (M. Cotlar, E. Stein). Let $\left(A_{j}\right)_{j \geq 1}$ be a family of Hilbert space operators such that

$$
\begin{gathered}
a=\sup _{i \geq 1} \sum_{j \geq 1}\left(\max \left(\left\|A_{i}^{*} A_{j}\right\|,\left\|A_{i} A_{j}^{*}\right\|\right)\right)^{1 / 2}<\infty, \\
b=\sup _{j \geq 1} \sum_{i \geq 1}\left(\max \left(\left\|A_{i}^{*} A_{j}\right\|,\left\|A_{i} A_{j}^{*}\right\|\right)\right)^{1 / 2}<\infty .
\end{gathered}
$$

Then the series $A x=\sum_{j} A_{j} x$ is unconditionally convergent for every $x \in H$, and $\|A\| \leq(a b)^{1 / 2}$.

Proof of Theorem 4.6. Suppose, to the contrary, that there exists $\delta$ with $0<\delta \leq 1$ such that $c_{1}(\delta$, $\operatorname{Mult}(\mathcal{X}))=\infty$, i.e.,

$$
c_{1}(\delta, \operatorname{Mult}(\mathcal{X}))=\sup \left\{\left\|T^{-1}\right\|: T \in \operatorname{Mult}(\mathcal{X}), \delta \leq\left|\lambda_{j}(T)\right| \leq\|T\| \leq 1, \forall j\right\}=\infty .
$$


This is equivalent to saying that

$$
\inf \left\{\|T x\|: x \in H,\|x\|=1, T \in \operatorname{Mult}(\mathcal{X}), \delta \leq\left|\lambda_{j}(T)\right| \leq\|T\| \leq 1, \forall j\right\}=0 .
$$

Without loss of generality, we may assume that $\left\|x_{j}\right\|=1$ for every $j$. Next, given an interval $J=(a, b)$, we denote by $P_{J}$ a projection defined by the basis decomposition $x=\sum_{j \geq 1} c_{j}(x) x_{j}$ as follows:

$$
P_{J} x=\sum_{j \in J} c_{j}(x) x_{j}
$$

Clearly, $P_{J}$ is a bounded operator and $\sup _{J}\left\|P_{J}\right\|<\infty$.

Our first observation is that, using the basis property

$$
\lim _{m \longrightarrow \infty} P_{[1, m)} T x=\lim _{m \longrightarrow \infty} T\left(P_{[1, m)} x\right)=T x \text { for every } x \in H,
$$

it is easy to show that

$$
\begin{aligned}
& \inf _{m \geq n} \inf \left\{\|T x\|: x \in P_{(n, m)} H,\|x\|=1,\right. \\
& \left.T \in \operatorname{Mult}(\mathcal{X}), \delta \leq\left|\lambda_{j}(T)\right| \leq\|T\| \leq 1 \text { for all } j\right\}=0
\end{aligned}
$$

for every $n=1,2, \ldots$.

Next, we observe that, for any finite interval $J^{\prime}$ and any number $\epsilon>0$, there exists $N$ such that

$$
\max \left(\left\|P_{J} P_{J^{\prime}}^{*}\right\|,\left\|P_{J}^{*} P_{J^{\prime}}\right\|,\left\|P_{J^{\prime}} P_{J}^{*}\right\|,\left\|P_{J^{\prime}}^{*} P_{J}\right\|\right)<\epsilon
$$

whenever $J=(a, b)$ with $a \geq N$. Indeed, since $P_{J^{\prime}}=\sum_{j \in J^{\prime}} P_{j}$, it suffices to verify the property for $P_{j}$, where $P_{j} x=c_{j}(x) x_{j}=\left(x, f_{j}\right) x_{j}, x \in H, f_{j}$ being the system biorthogonal to $\left(x_{j}\right)$ (which is also a basis). However, $\left\|P_{J} P_{j}^{*}\right\|=\left\|P_{J}\left(\cdot, x_{j}\right) f_{j}\right\|=$ $\left\|x_{j}\right\| \cdot\left\|P_{J} f_{j}\right\|=\left\|P_{J} f_{j}\right\|$, which tends to zero as $N \longrightarrow 0$ (an easy basis property). As to $P_{j} P_{J}^{*}$, it is simply zero, $P_{j} P_{J}^{*}=0$ when $N>j$. The quantities $\left\|P_{J}^{*} P_{J^{\prime}}\right\|$ and $\left\|P_{J^{\prime}}^{*} P_{J}\right\|$ can be treated similarly (or by exchanging the bases $\left(x_{j}\right)$ and $\left(f_{j}\right)$ ). This shows the property claimed.

Using the above observations, we construct a sequence of pairwise disjoint finite intervals $\left(J_{k}\right)_{k \geq 1}$ in the following two-step induction process:

1) first, we choose a sufficiently large integer $a$ such that, for every $J_{k}=(a, b)$,

$$
\max \left(\left\|P_{J_{k}} P_{J_{i}}^{*}\right\|,\left\|P_{J_{k}}^{*} P_{J_{i}}\right\|,\left\|P_{J_{i}} P_{J_{k}}^{*}\right\|,\left\|P_{J_{i}}^{*} P_{J_{k}}\right\|\right)<2^{-k}
$$

for all $i, 1 \leq i<k$, and

2) next, we find $b$ so large that

$\inf \left\{\|T x\|: x \in P_{J_{k}} H,\|x\|=1, T \in \operatorname{Mult}(\mathcal{X}), \delta \leq\left|\lambda_{j}(T)\right| \leq\|T\| \leq 1\right.$ for all $\left.j\right\}<1 / k$.

Therefore, for every $k$, there exists a multiplier $T_{k}$ and a vector $x_{k} \in P_{J_{k}} H$ such that $\delta \leq\left|\lambda_{j}\left(T_{k}\right)\right| \leq\left\|T_{k}\right\| \leq 1$ for all $j$, and $\left\|x_{k}\right\|=1,\left\|T_{k} x_{k}\right\|<1 / k$.

Now, we apply Cotlar-Stein's lemma to ensure that the series

$$
T=\left(I-\sum_{k} P_{J_{k}}\right)+\sum_{k} T_{k} P_{J_{k}}
$$

converges to a bounded operator, which is obviously a multiplier satisfying $\delta \leq\left|\lambda_{j}(T)\right|$ for every $j$, and $\left\|T x_{k}\right\|=\left\|T_{k} x_{k}\right\|<1 / k$ for every $k$. This contradicts the SLP property of $\operatorname{Mult}(\mathcal{X})$.

A similar application of Cotlar-Stein's lemma shows the following property.

4.7. Theorem. There exists no sequence $\mathcal{X}=\left\{x_{j}\right\}_{j \geq 1}$ in a Hilbert space $H$ such that $\operatorname{Mult}(\mathcal{X})=b v$. 
Proof. Assuming $b v \subset \operatorname{Mult}(\mathcal{X})$, we know that $\mathcal{X}$ is a basic sequence. Applying the same procedure as in the proof of Theorem 4.6, we see that the operator

$$
T=\left(I-\sum_{k} P_{J_{k}}\right)+\sum_{k} a_{k} P_{J_{k}}
$$

is a multiplier of $\mathcal{X}$ whatever a sequence $\left(a_{k}\right) \in l^{\infty}$ is. Taking $a_{k}=(-1)^{k}$, we get $\sum_{j \geq 1}\left|\lambda_{j}(T)-\lambda_{j+1}(T)\right|=\infty$, but $T \in \operatorname{Mult}(\mathcal{X})$. Therefore, $\operatorname{Mult}(\mathcal{X}) \neq b v$.

We finish this discussion of links between the SLP and the critical constant with the following observation. Recall that a sequence $\mathcal{X}=\left(x_{j}\right)$ in a Banach space $X$ is minimal if and only if there exists a sequence of biorthogonal functionals $f_{k} \in X^{*}$ such that $\left\langle x_{j}, f_{k}\right\rangle=\delta_{j k}$ for every $j$ and $k$. Surely, every basic sequence is minimal.

4.8. Theorem. Let $\mathcal{X}=\left(x_{j}\right)_{j \geq 1}$ be a minimal sequence in a Banach space $X$. The following statements are equivalent:

1) $\mathcal{X}$ is an unconditional basic sequence;

2) $\delta_{1}(\mathcal{X})=0$ and $c_{1}(\delta, \mathcal{X}) \leq C / \delta$ for every $0<\delta \leq 1$ (in particular, $\mathcal{X} \in S L P$ ).

Proof. Since 1) implies $\operatorname{Mult}(\mathcal{X})=l^{\infty}$, it is clear that 1) $\Longrightarrow 2$ ).

Assuming 2), we take a finite subset $\sigma \subset \mathbb{N}$ and a number $a>1$ and define a multiplier $T_{a}$ by

$$
T_{a} x_{j}=x_{j} \text { for } j \in \sigma, \text { and } T_{a} x_{j}=a x_{j} \text { for } j \in \mathbb{N} \backslash \sigma .
$$

This is a multiplier because $\mathcal{X}$ is a minimal sequence. Moreover,

$$
\lambda_{j}\left(\frac{T_{a}}{\left\|T_{a}\right\|}\right) \geq \delta=1 /\left\|T_{a}\right\|
$$

for every $j$, whence $\left\|T_{a}^{-1}\right\| \cdot\left\|T_{a}\right\| \leq C\left\|T_{a}\right\|$, i.e., $\left\|T_{a}^{-1}\right\| \leq C$ for every $a>1$. Letting $a$ tend to $\infty$, we see that the projection $P_{\sigma}, P_{\sigma} x_{j}=x_{j}$ for $j \in \sigma$ and $P_{\sigma} x_{j}=0$ for $j \in \mathbb{N} \backslash \sigma$, is bounded by $C:\left\|P_{\sigma}\right\| \leq C$. This shows that $\mathcal{X}$ is an unconditional basic sequence.

\section{§5. Miscellanea: A corona problem, gap measures, SlP for semigroups}

5.1. The multiplier corona problem. This problem was already mentioned in the Introduction. In general, the "corona property", i.e., the density of $J$ in $\mathfrak{M}\left(\operatorname{Mult}\left(\left(x_{j}\right)_{j \in J}\right)\right)$, is a much stronger property than the SLP, but sometimes they coincide.

5.1.1. Proposition. Assume $\operatorname{Mult}(\mathcal{X}), \mathcal{X}=\left(x_{j}\right)_{j \in J}$, is a conjugate symmetric space, that is, $A=\left(\lambda_{j}\right) \in \operatorname{Mult}(\mathcal{X})$ implies that $\bar{A}=\left(\bar{\lambda}_{j}\right) \in \operatorname{Mult}(\mathcal{X})$. Then the SLP entails the "corona property" $\operatorname{clos\mathfrak {M}}(J)=\mathfrak{M}(\operatorname{Mult}(\mathcal{X}))$.

Proof. As is well known, the corona property is equivalent to the solvability of the Bézout equations for columns $T^{\mathrm{col}}=\left(T_{k}\right)_{k=1}^{n} \in \operatorname{Mult}(\mathcal{X})^{n}$, i.e., to the existence of a row $S \in$ $\operatorname{Mult}(\mathcal{X})^{n}$ such that

$$
S T^{\mathrm{col}}=\sum_{k=1}^{n} S_{k} T_{k}=I d
$$

provided a necessary condition is fulfilled:

$$
\delta=\inf _{j}\left|\lambda_{j}(T)\right|>0,
$$

where $\left|\lambda_{j}(T)\right|=\left(\sum_{k=1}^{n}\left|\lambda_{j}\left(T_{k}\right)\right|^{2}\right)^{1 / 2}$. By the hypothesis, we have $\lambda_{j}\left(\bar{T}_{k}\right)=\bar{\lambda}_{j}\left(T_{k}\right)$, whence $\lambda_{j}(A)=\sum_{k=1}^{n} \lambda_{j}\left(T_{k}\right) \bar{\lambda}_{j}\left(T_{k}\right)=\sum_{k=1}^{n}\left|\lambda_{j}\left(T_{k}\right)\right|^{2}, j \in J$, are multipliers, and obviously $\inf _{j} \lambda_{j}(A)>0$. The SLP implies $A^{-1} \in \operatorname{Mult}(\mathcal{X})$, so that the $S_{k}=A^{-1} \bar{T}_{k}$ solve the equation. 
5.1.2. Corollary. If a measure $\mu$ on $\mathbb{T}$ is "almost conjugate symmetric" in the sense that $\mu(\bar{A})=: \mu_{*}(A) \leq c \mu(A)$ for every Borel subset $A \subset \mathbb{T}$, then the $S L P$ for $\operatorname{Mult}\left(\mathcal{E}, L^{2}(\mathbb{T}, \mu)\right)$ implies the "corona property".

Indeed, it suffices to verify the hypothesis of Proposition 5.1.1. For this, we take a multiplier $T=\left(\lambda_{j}\right)_{j \in \mathbb{Z}}$ and a trigonometric polynomial $p=\sum_{j} a_{j} z^{j}$ and use the notation $p_{*}=\sum_{j} \bar{a}_{j} z^{j}$ to write

$$
\begin{aligned}
& \int_{\mathbb{T}} \mid \bar{T}\left.p(z)\right|^{2} d \mu(z)=\int_{\mathbb{T}}\left|\sum_{j} \bar{\lambda}_{j} a_{j} z^{j}\right|^{2} d \mu(z)=\int_{\mathbb{T}}\left|\sum_{j} \lambda_{j} \bar{a}_{j} \bar{z}^{j}\right|^{2} d \mu(z) \\
&= \int_{\mathbb{T}}\left|\sum_{j} \lambda_{j} \bar{a}_{j} z^{j}\right|^{2} d \mu_{*}(z)=\int_{\mathbb{T}}\left|T p_{*}(z)\right|^{2} d \mu_{*}(z) \leq c \int_{\mathbb{T}}\left|T p_{*}(z)\right|^{2} d \mu(z) \\
& \leq c\|T\|^{2} \int_{\mathbb{T}}\left|p_{*}(z)\right|^{2} d \mu(z)=c\|T\|^{2} \int_{\mathbb{T}}|p(z)|^{2} d \mu_{*}(z) \\
& \leq c^{2}\|T\|^{2} \int_{\mathbb{T}}|p(z)|^{2} d \mu(z) .
\end{aligned}
$$

This shows that $\bar{T}$ is a multiplier.

5.1.3. Remark. One can easily observe that for every measure $\mu$ we have $\left(\mu+\mu_{*}\right)_{*}=$ $\mu+\mu_{*}$. On the other hand, for nonsymmetric algebras, the SLP does not imply the "corona property". For example, starting with a B. Cole example (see G]) of a Riemann surface $\Omega$ for which the algebra $H^{\infty}(\Omega)$ has a corona, we can proceed as in Example 4.2 above in order to obtain a sequence $\mathcal{X}$ of reproducing kernels of the Hardy space $H^{2}(\Omega)$ such that $\operatorname{Mult}(\mathcal{X})$ satisfies the SLP but has a corona.

5.2. Gap measures, and other instabilities in $\operatorname{Mult}\left(\mathcal{E}, L^{2}(\mathbb{T}, \mu)\right)$. Here, we exhibit two examples showing how (even tiny) perturbations of a measure $\mu$ could dramatically change the multiplier algebra $\operatorname{Mult}(\mu)=\operatorname{Mult}\left(\mathcal{E}, L^{2}(\mathbb{T}, \mu)\right)$ and its SLP.

5.2.1. Gap measures. Let $\mu$ be a measure having as its closed support the entire circle $\mathbb{T}$ with an arc gap: $\operatorname{supp}(\mu)=\mathbb{T} \backslash \gamma$, where $\gamma \subset \mathbb{T}$ is a nonempty open arc. For instance, we can think about this setting as if $\mu=\mu_{0}-\mu_{0} \chi_{\gamma}$, where $\operatorname{supp}\left(\mu_{0}\right)=\mathbb{T}$. In this case, surprisingly, there are no nontrivial multipliers. Namely, we have the following proposition.

5.2.2. Proposition. Assume that the support of a measure $\mu$ on $\mathbb{T}, \mu \neq 0$, has a nontrivial arc gap: $\operatorname{supp}(\mu)=\mathbb{T} \backslash \gamma, \gamma$ being an open arc $\gamma \neq \varnothing$. Then $\operatorname{Mult}(\mu)=\mathbb{1} \cdot \mathbb{C}$, where $\mathbb{1}=\{1\}$ (the constant sequence). In particular, $\mathcal{E}$ in $L^{2}(\mathbb{T}, \mu)$ satisfies the SLP.

Proof. Since $\operatorname{Mult}(\mu)=\operatorname{Mult}\left(\tau_{\zeta} \mu\right)$ for every $\zeta \in \mathbb{T}$, where $\tau_{\zeta} \mu$ stands for a rotation of $\mu\left(\tau_{\zeta} \mu(A)=\mu(\bar{\zeta} A)\right.$ for $\left.\mathrm{A} \subset \mathbb{T}\right)$, we can assume that $\gamma$ is a symmetric (closed) arc that contains 1 and is of the form $\gamma=[\alpha, \bar{\alpha}], \alpha=e^{i \theta}, 0 \leq \theta<\pi$. We also can assume without loss of generality that $\mathbb{T} \backslash \gamma$ is not a point (if not, $\operatorname{dim} L^{2}(\mu)=1$, and the result follows).

Let $T=\left(\lambda_{j}\right)$ be a multiplier of $L^{2}(\mu)$. As an operator on $L^{2}(\mu), T$ is convolution with a distribution $t=\sum_{j \in \mathbb{Z}} \lambda_{j} z^{j}$ (in fact, a pseudomeasure, because $\sup _{j}\left|\lambda_{j}\right|<\infty$ ).

We show that $\operatorname{supp}(t)=\{1\}$. Supposing the contrary, we can find $\zeta \in \gamma$ such that

$$
\left(\operatorname{supp}\left(\delta_{\zeta} * t\right)\right) \cap(\mathbb{T} \backslash \bar{\gamma})=\left(\tau_{\zeta}(\operatorname{supp}(t))\right) \cap(\mathbb{T} \backslash \bar{\gamma}) \neq \varnothing .
$$

(Indeed, if $\lambda \in \mathbb{T} \backslash\{1\}$, then either $\lambda \in \mathbb{T} \backslash \bar{\gamma}$ and we can choose $\zeta=1$, or $\lambda \in \bar{\gamma}$ and an obvious geometric argument shows that, in this case too, there exists $\zeta \in \gamma$ such that $\bar{\zeta} \lambda \in \mathbb{T} \backslash \bar{\gamma}$.) Let $\left(\varphi_{n}\right)$ be a $C^{\infty}$ approximate identity on $\mathbb{T}$ having supports that converge to 1 , and let $\psi_{n}=\delta_{\zeta} * \varphi_{n}$. Then there exists $n$ such that $\operatorname{supp}\left(\psi_{n}\right) \subset \gamma$ 
and $\left(\operatorname{supp}\left(\psi_{n} * t\right)\right) \cap(\mathbb{T} \backslash \bar{\gamma}) \neq \varnothing$. The latter property implies that the $C^{\infty}$-function $T \psi_{n}=\psi_{n} * t$ is not zero in $L^{2}(\mu)$, whereas the former inclusion shows that $\psi_{n}=0$ in $L^{2}(\mu)$. This means that $T$ is not well defined, which is absurd. Hence, $\operatorname{supp}(t)=\{1\}$.

But it is well known that a pseudomeasure with finite support is a measure (see, for instance, $[\mathrm{KS}]$ ). Hence, $t=c \delta_{1}$, where $c \in \mathbb{C}$, as claimed.

5.2.3. Remark. It is curious to note that in the case where the support gap $\mathbb{T} \backslash \operatorname{supp}(\mu)$ is not empty but contains more than one arc, the result of Proposition 5.2.2 is false. Indeed, it is easily seen that for $\mu=\delta_{1}+\delta_{-1}$, the algebra Mult $(\mu)$ consists of all 2periodic sequences on $\mathbb{Z}$.

Yet another "instability" result is as follows.

5.2.4. Proposition. Let $\mu=m+a \delta_{1}$, where $m$ stands for the normalized Lebesgue measure on $\mathbb{T}$ and $a>0$. Then $\operatorname{Mult}(\mu)=l^{2}+\mathbb{1} \cdot \mathbb{C}$; in particular, $\mathcal{E}$ in $L^{2}(\mathbb{T}, \mu)$ satisfies the SLP.

Proof. First, we show that $\operatorname{Mult}(\mu) \subset l^{2}+\mathbb{1} \cdot \mathbb{C}$. To this end, let $T=\left(\lambda_{j}\right)$ be a multiplier of $\mathcal{E}$ on $L^{2}(\mathbb{T}, \mu)$. Then

$$
\int_{\mathbb{T}}|T p|^{2} d \mu \leq a|T p(1)|^{2}+\int_{\mathbb{T}}|T p|^{2} d m \leq\|T\|^{2} \int_{\mathbb{T}}|p|^{2} d \mu .
$$

In particular, making $p$ explicit, $p=\sum_{j} c_{j} z^{j}$, we obtain

$$
\left|\sum_{j} \lambda_{j} c_{j}\right|^{2} \leq a^{-1}\|T\|^{2} \int_{\mathbb{T}}|p|^{2} d \mu
$$

for every polynomial $p$. Therefore, $\left|\sum_{j} \lambda_{j} c_{j}\right|^{2} \leq a^{-1}\|T\|^{2} \int_{\mathbb{T}}|p|^{2} d m$ for every polynomial $p$ with $p(1)=0$. This means that the linear form $p \longmapsto \sum_{j} \lambda_{j} c_{j}$ is bounded on the linear manifold $L=\{p: p(1)=0\}$. But $L$ is dense in $L^{2}(m)$, so that this form (uniquely) extends to a bounded form on $L^{2}(m)$. So, there exists a sequence $\left(b_{j}\right) \in l^{2}$ such that $\sum_{j} \lambda_{j} c_{j}=\sum_{j} b_{j} c_{j}$ for every polynomial $p$ with $p(1)=\sum_{j} c_{j}=0$. This means that there is $b \in \mathbb{C}$ such that $\lambda_{j}=b_{j}+b$ for every $j \in \mathbb{Z}$. The inclusion follows.

The reverse inclusion is obvious because, in the case of $T=\left(b_{j}+b\right),\left(b_{j}\right) \in l^{2}$, we have

$$
\begin{aligned}
\int_{\mathbb{T}}|T p|^{2} d \mu & =\int_{\mathbb{T}}|T p|^{2} d m+a\left|\sum_{j} b_{j} c_{j}+b p(1)\right|^{2} \\
& \leq A \int_{\mathbb{T}}|p|^{2} d m+2 a\left|\sum_{j} b_{j} c_{j}\right|^{2}+2 a|b p(1)|^{2} \\
& \leq A \int_{\mathbb{T}}|p|^{2} d m+B \int_{\mathbb{T}}|p|^{2} d m+2 a|b|^{2} \int_{\mathbb{T}}|p|^{2} d \delta_{1} \leq C \int_{\mathbb{T}}|p|^{2} d \mu
\end{aligned}
$$

for every polynomial $p$; thus, $T$ is a multiplier.

5.3. The spectral localization property for $C_{0}$ semigroups. The SLP problem for semigroups is similar to that for a single operator: given a family of vectors $\mathcal{X}=\left(x_{j}\right)_{j \in J}$ in a Banach space $X$, we say that the semigroup spectral localization property (SSLP) holds for $\mathcal{X}$ if for any $C_{0}$ semigroup $S(t), t>0$, on $\operatorname{span}_{X}(\mathcal{X})$ such that

$$
S(t) x_{j}=\lambda_{j}(t) x_{j} \text { for all } j \text { and all } t>0,
$$

we have

$$
\sigma(S(t))=\operatorname{clos}\left\{\lambda_{j}(t)\right\}
$$

for every $t>0$. Clearly, the SLP for $\mathcal{X}$ implies the SSLP for $\mathcal{X}$. In particular, any unconditional basis, or a sequence with $\operatorname{Mult}(\mathcal{X})=b v, l^{2}+\mathbb{1} \cdot \mathbb{C}$, or $\mathbb{1} \cdot \mathbb{C}$, etc., satisfies 
the SSLP. The following two examples describe the only nontrivial information we have concerning the SSLP.

5.3.1. Proposition. Let $H=L^{2}\left(\mathbb{R}_{+}\right)$, and let $\mathcal{X}=\left(e_{j}\right)_{j \in \mathbb{Z}}$ be a family of functions in $H, e_{j}(x)=e^{i \gamma_{j} x}\left(x \in \mathbb{R}_{+}\right)$, where

$$
0<\alpha=\inf _{j} \operatorname{Im}\left(\gamma_{j}\right) \leq \sup _{j} \operatorname{Im}\left(\gamma_{j}\right)=\beta<\infty .
$$

The following statements are equivalent:

1) $\mathcal{X} \in S S L P$;

2) $\mathcal{X} \in S L P$

3) $\left(\gamma_{j}\right)$ has finite Carleson density

$$
\sup _{x \in \mathbb{R}, l \geq 1} \frac{\operatorname{card}\left\{j: x \leq \operatorname{Re}\left(\gamma_{j}\right) \leq x+l\right\}}{l}<\infty .
$$

In the case where $\mathcal{X} \notin S S L P$, a semigroup $S$ giving a counterexample (such that $\inf _{j}\left|\lambda_{j}(S(t))\right|>0$ but $0 \in \sigma(S(t))$ for every $\left.t>0\right)$ is simply the translation semigroup $S(t) f(x)=f(x+t), t>0, f \in \operatorname{span}_{H} \mathcal{X}$.

In particular, the numbers $\gamma_{j}=i+j|j|^{\alpha-1}(j \in \mathbb{Z}), 1 / 2<\alpha<1$, form a Blaschke sequence NOT satisfying 3 ), and hence, the corresponding sequence $\mathcal{X}$ is a summation basis not satisfying the SSLP.

Proof. Condition 3) implies 2) (and hence 1)) due to [GMN] (see Subsection 4.3 above), because condition 3$)$ means that $\left(\gamma_{j}\right)$ is a finite union of interpolating sequences for the algebra $H^{\infty}\left(\mathbb{C}_{+}\right)$of the half-plane $\mathbb{C}_{+}=\{z \in \mathbb{C}: \operatorname{Im}(z)>0\}$ (see [Ni1, vol. 2, p. 303]).

It remains to show that 1) $\Longrightarrow 3$ ). For this, we assume that 3 ) is not satisfied and, hence, for every $N>0$ there exists $x \in \mathbb{R}$ such that $\operatorname{card}\left\{j: \operatorname{Re}\left(\gamma_{j}\right) \in I=[x, x+1]\right\}>N$. Now, we can check that the functions

$$
f_{t}(z)=e^{i t z}
$$

are not invertible in the quotient algebra $H^{\infty}\left(\mathbb{C}_{+}\right) / B H^{\infty}\left(\mathbb{C}_{+}\right)$, where $B$ stands for the Blaschke product $B=\prod_{j} b_{\gamma_{j}}, b_{\mu}=\epsilon \frac{z-\mu}{z-\bar{\mu}}, \operatorname{Im}(\mu)>0,|\epsilon|=1$. (Without loss of generality, the Blaschke product converges; if not, the argument is similar but even easier (take a partial product instead of $B$ ).) It suffices to show that

$$
\inf _{\operatorname{Im}(z)>0}\left(\left|f_{t}(z)\right|+|B(z)|\right)=0 .
$$

Taking $z_{N}=x+i N / \log N=x+i y$, we get

$$
\left|B\left(z_{N}\right)\right|^{2} \leq \prod_{\gamma_{j} \in I}\left|\frac{z_{N}-\gamma_{j}}{z_{N}-\bar{\gamma}_{j}}\right|^{2} \leq \prod_{\gamma_{j} \in I}\left|\frac{a_{j}^{2}+(y-\alpha)^{2}}{a_{j}^{2}+(y+\alpha)^{2}}\right|^{2},
$$

where $a_{j}^{2} \leq 1$. Hence,

$$
\left|B\left(z_{N}\right)\right|^{2} \leq \prod_{1}^{N}\left|\frac{1+(y-\alpha)^{2}}{1+(y+\alpha)^{2}}\right|^{2}=\left(1-\frac{4 \alpha y}{1+(y+\alpha)^{2}}\right)^{N}
$$

(assuming, without loss of generality, that $1+2 \alpha y+\alpha^{2} \leq y^{2}$ )

$$
\leq\left(1-\frac{4 \alpha y}{2 y^{2}}\right)^{N}=\left(1-\frac{2 \alpha \log N}{N}\right)^{N},
$$

which tends to zero as $N \longrightarrow \infty$. Obviously, $\lim _{N}\left|f_{t}\left(z_{N}\right)\right|=0$ for every $t>0$. Therefore, $f_{t}$ is not invertible in $H^{\infty}\left(\mathbb{C}_{+}\right) / B H^{\infty}\left(\mathbb{C}_{+}\right)$, and hence $S(t) \mid H$ is not invertible (for more 
details, see [Ni4, p. 66], or [Ni1, vol. 2, p. 103], or [GMN]), whereas

$$
\left|\lambda_{j}(S(t))\right|=\left|f_{t}\left(\gamma_{j}\right)\right| \geq e^{-t \beta}
$$

for every $j$. This contradicts property 1 ).

For the fact that every sequence of Szegö reproducing kernels corresponding to a Blaschke sequence is a summation basis, see [Ni1, vol. 2, p. 181] or [Ni4, p. 194].

From Proposition 5.3.1 we can derive the following example.

5.3.2. Example: a summation basis in a Hilbert space, which is finitely block-orthogonal and does not satisfy the SSLP. The construction is similar to those of Example 4.4, so we give a draft only. Namely, starting with an example from Proposition 5.3.1, we cut the spectrum $\left(\gamma_{j}\right)$ in the pieces $n_{k} \leq j<n_{k+1}$, where $n_{k} \uparrow \infty$. Put

$$
\begin{aligned}
H_{k} & =\operatorname{span}\left(x_{j}: n_{k} \leq j<n_{k+1}\right), \\
H & =\sum_{k \geq 1} \oplus H_{k}
\end{aligned}
$$

and

$$
s(t)=\sum_{k \geq 1} \oplus S(t) \mid H_{k}
$$

for $t>0$. If $\left(n_{k}\right)$ grows sufficiently fast, we obtain the required properties of $\left(x_{j}\right)$ and

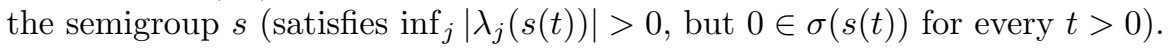

\section{REFERENCES}

[D] J. Duoandikoetxea, Fourier analysis, Grad. Stud. in Math., vol. 29, Amer. Math. Soc., Providence, RI, 2001. MR1800316 (2001k:4200)

[ENZ] O. El-Fallah, N. K. Nikolski, and M. Zarrabi, Resolvent estimates in Beurling-Sobolev algebras, Algebra i Analiz 10 (1998), no. 6, 1-92; English transl., St. Petersburg Math. J. 10 (1999), no. 6, 901-964. MR1678988 (2000c:46101)

[G] T. Gamelin, Uniform algebras and Jensen measures, London Math. Soc. Lecture Note Ser., vol. 32, Cambridge Univ. Press, Cambridge-New York, 1978. MR0521440 (81a:46058)

[GMN] P. Gorkin, R. Mortini, and N. Nikolski, Norm controlled inversions and a corona theorem for $H^{\infty}$-quotient algebras, J. Funct. Anal. 255 (2008), 854-876. MR2433955 (2009h:30061)

[GMcG] C. C. Graham and O. C. McGehee, Essays in commutative harmonic analysis, Grundlehren Math. Wiss., Bd. 238, Springer-Verlag, New York-Berlin, 1979. MR0550606 (81d:43001)

[KS] J. -P. Kahane and R. Salem, Ensembles parfaits et séries trigonométriques, Actualités Sci. Indust., No. 1301, Hermann, Paris, 1963. MR0160065 (28:3279)

[Ni1] N. Nikolski, Operators, functions, and systems. Vols. 1, 2, Math. Surveys Monogr., vols. 92, 93, Amer. Math. Soc., Providence, RI, 2002. MR1864396 (2003i:47001a) MR1892647 (2003i:47001b)

[Ni2] In search of the invisible spectrum, Ann. Inst. Fourier (Grenoble) 49 (1999), 1925-1998. MR.1738071 (2001a:46053)

[Ni3] The problem of efficient inversions and Bézout equations, Twentieth Century Harmonic Analysis - a Celebration (Il Ciocco, 2000), NATO Sci. Ser. II Math. Phys. Chem., vol. 33, Kluwer Acad. Publ., Dordrecht, 2001, pp. 235-269. MR.1858788(2003a:43004)

[Ni4] Lectures on the shift operator, Nauka, Moscow, 1980; English transl., Treatise on the shift operator. Spectral function theory, Grundlehren Math. Wiss., Bd. 273, Springer-Verlag, Berlin, 1986. MR0575166 (82i:47013) MR0827223 (87i:47042)

[NV] V. Vasyunin and N. Nikolski, Algebras of traces of algebras $H^{\infty}$ with a given critical constant. Application to Bourgain-Tzafriri problem (in preparation). (Russian)

[R] W. Rudin, Fourier analysis on groups, Intersci. Tracts in Pure Appl. Math., No. 12, Intersci. Publ., New York-London, 1962. MR0152834 (27:280)

[Si] I. Singer, Bases in Banach spaces. I, II; I, Grundlehren Math. Wiss., Bd. 154, Springer-Verlag, New York-Berlin, 1970; II, Springer-Verlag, Berlin-New York, 1981. MR0298399(45:7451) MR0610799 (82k:46024) 
[St] E. Stein, Harmonic analysis: real-variable methods, orthogonality, and oscillatory integrals, Princeton Math. Ser., vol. 43. Monogr. in Harmonic Anal., III, Princeton Univ. Press, Princeton, NJ, 1993. MR1232192 (95c:42002)

[T] A. Torchinsky, Real-variable methods in harmonic analysis, Pure Appl. Math., vol. 123, Acad. Press, Inc., Orlando, FL, 1986. MR0869816 (88e:42001)

Université de Bordeaux 1, UFR de Mathématiques et Informatique, 351 Cours de la Libération, 33405, Talence, France, and St. Petersburg Branch, Steklov Mathematical Institute, Fontanka 27, St. Petersburg 191023, Russia

E-mail address: nikolski@math.u-bordeaux1.fr

Received 18/AUG/2009

Originally published in English 